\title{
Correction to: Appendectomy versus conservative treatment with antibiotics for patients with uncomplicated acute appendicitis: a propensity score-matched analysis of patient-centered outcomes (the ACTUAA prospective multicenter trial)
}

\author{
Mauro Podda ${ }^{1,2}$ (D) $\cdot$ Gaetano Poillucci $^{3}$ - Daniela Pacella ${ }^{4}$ - Lorenzo Mortola ${ }^{5}$ - Alfonso Canfora ${ }^{6} \cdot$ Simona Aresu $^{7}$.

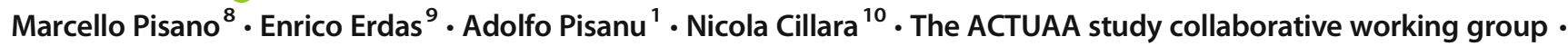 \\ Fernando Serventi • Stefano Marini • Danilo Sirigu • Michela Piga • Massimiliano Coppola • Francesco Balestra • \\ Carlo De Nisco - Marco Pazzona - Marco Anania • Fabio Pulighe • Antonio Lai • Roberto Ottonello • \\ Renato De Angelis • Silverio Piro • Pietro Giorgio Calò • Francesco Podda • Luca Saba • Vincenzo Bottino • \\ Patrizia Dalla Caneva • Luisa Canu • Emanuele Piras • Antonello Deserra • Francesco Virdis • Chiara Gerardi • \\ Luca Gordini · Silvia Sanna
}

Published online: 28 January 2021

(C) Springer-Verlag GmbH Germany, part of Springer Nature 2021

Correction to: International Journal of Colorectal Disease https://doi.org/10.1007/s00384-021-03843-8

All authors and affiliations of the The ACTUAA Study Collaborative Working Group are listed in the Acknowledgments section of the original published version of this article however, Luca Gordini and Silvia Sanna should have been added in the list. Thus, the names are now presented and cited correctly. The original article has been corrected.

Publisher's note Springer Nature remains neutral with regard to jurisdictional claims in published maps and institutional affiliations.

The online version of the original article can be found at https://doi.org/ $10.1007 / \mathrm{s} 00384-021-03843-8$

Mauro Podda

mauropodda@ymail.com

1 Department of Emergency Surgery, Cagliari University Hospital

"Duilio Casula", Azienda Ospedaliero-Universitaria di Cagliari, Cagliari, Italy

2 Policlinico Universitario di Monserrato "Duilio Casula", University of Cagliari, SS 554, Km 4,500, 09042 Monserrato, Italy

3 Department of General and Specialized Surgery "Paride Stefanini", Policlinico Universitario Umberto I, La Sapienza University, Rome, Italy

4 Department of Public Health, University of Naples Federico II, Naples, Italy

5 Department of Surgery, Azienda Ospedaliero-Universitaria Maggiore della Carità, Novara, Italy

Department of Surgery, Villa Betania Hospital, Naples, Italy

7 Department of Surgery, Nostra Signora della Mercede Hospital, Lanusei, Italy

8 Department of Surgery, San Marcellino Hospital, ASSL Cagliari, Muravera, Italy

9 Department of General and Endocrine Surgery, Cagliari University Hospital "Duilio Casula", Azienda Ospedaliero-Universitaria di Cagliari, Cagliari, Italy

10 Department of General Surgery, Santissima Trinità Hospital, ASSL Cagliari, Cagliari, Italy 